\title{
Evaluation of an Interprofessional Academic-Practice Partnership in End of Life Care
}

Katie F. Leslie, PhD, MS ; M. Kate Probst, PharmD²; Taylor L. Hawkins, PharmD ${ }^{3}$

${ }^{1}$ Sullivan University College of Pharmacy and Health Sciences; ${ }^{2}$ Hosparus Health; ${ }^{3}$ Baptist Health Louisville

\begin{abstract}
Introduction: The purpose of this mixed-methods study was to evaluate an interprofessional academic-practice partnership in end of life care by examining patient medication outcomes, the contributions of student pharmacists and a pharmacy preceptor to care teams, and student learning experiences.

Methods: Retrospective chart review assessed polypharmacy differences in hospice patients with a primary terminal diagnosis of nonAlzheimer's dementia between two patient groups; Group 1 managed on interprofessional care teams within the pharmacy partnership, and Group 2, managed on teams without a pharmacist. Team members who interacted with student pharmacists and the pharmacy preceptor participated in semi-structured key informant interviews to document perceptions of pharmacy contributions to care teams and the organization. At the end of their APPE, students completed reflective writings regarding their learning.

Results: Patients in Group 1 were on statistically significant fewer medications than Group 2 at both week 4 and weeks 7-12 following admission. Five conceptual themes emerged from interviews: pharmacists as team medication experts, improved patient outcomes, interprofessional collaboration, patient/caregiver trust in medication regimens, and desire for sustainability. Student reflections included the following learning themes: teamwork, respect, value, and patient-centered care.

Conclusions: The addition of a pharmacist on interprofessional care teams decreased the average number of medications in the nonAlzheimer's end of life patient population. Team members identified value-added contributions of student pharmacists and the pharmacy preceptor that enhanced team efficiency and patient care. Student pharmacists recognized these contributions and the experience served as an exemplar of interprofessional practice.
\end{abstract}

Keywords: Scholarship of Teaching and Learning, Palliative Care, Mixed Methods

\section{Introduction}

While varying among organizations and settings, pharmacists provide essential services to palliative and end of life interprofessional care teams. ${ }^{1}$ As patients transition from an aggressive to comfort-focused treatment models, pharmacists may assess medication regimens and initiate deprescribing interventions that are patient-focused, cost-effective, and evidence-based. ${ }^{1,2}$ Deprescribing is "the systematic process of identifying and discontinuing drugs in instances in which existing or potential harms outweigh existing or potential benefits within the context of an individual patient's care goals, current level of functioning, life expectancy, values, and preferences." ${ }^{3}$ Polypharmacy in advanced age is associated with higher symptom burden and lower quality of life, which is in direct opposition to the hospice mission of comfort-based care. ${ }^{4}$ The goal of deprescribing is to reduce polypharmacy and improve health outcomes, including quality of life measures. ${ }^{5,6}$ While there are known benefits of deprescribing, many barriers impede this in practice. ${ }^{7}$ Deprescribing is a complex and patient-specific process that requires specialized clinicians, communication and collaboration among providers, and a great amount of time dedicated to each patient. ${ }^{8-10}$

Corresponding author: Katie F. Leslie, PhD, MS

Associate Professor, Sullivan University

College of Pharmacy and Health Sciences

2100 Gardiner Ln., Louisville, KY 40205, USA

Phone: 502-777-1667; Email: kleslie@sullivan.edu
Deprescribing has gained increased attention in health sciences education with multiple calls for added content within curricula emphasizing opportunities for interprofessional learning experiences. ${ }^{11-14}$ Specific to academic pharmacy, there is significant need to enhance teaching of optimal prescribing and deprescribing related content with student assessment measures in the didactic and experiential curricula. ${ }^{12,15}$ In addition to educational content and evaluations, there are needs for further research into pharmacists' impact on deprescribing in end-of-life care settings, and to assess the need for and sustainability of palliative and hospice care pharmacists. $^{1}$

Within the American Association of Colleges of Pharmacy (AACP) Strategic Plan, academic-practice partnerships that enhance pharmacy education and promote interprofessional practice have been identified for further investigation. ${ }^{16}$ Partnerships of any type between colleges of pharmacy and clinical practice sites may ease financial, labor, time, and logistical constraints in many pharmacy practice settings. ${ }^{17}$ This allows for innovations in pharmacy practice, advancement of interprofessional education and practice, improved outcomes for health system partner organizations, and advancement of the mission and learning opportunities for the academic partner. ${ }^{17-19}$ However, there is a dearth of literature examining the impact of these partnerships from multiple stakeholder perspectives. The purpose of this study was to evaluate an interprofessional academic-practice partnership in end of life care that examined both student learning and the value- 
added contributions of student pharmacists and pharmacy preceptors.

\section{Methods}

This study assessed an academic-practice partnership surrounding an Advanced Pharmacy Practice Experience (APPE) in end of life care offered at a 3-year accelerated doctor of pharmacy (PharmD) program at a private college of pharmacy. The partnership began in March 2018 between the college of pharmacy and a local hospice organization. Prior to partnership initiation, all hospice pharmacy services at the partner organization were under an offsite, mail order pharmacy for dispensing, formulary determination, and other drug information requests, without direct pharmacist participation and input in interprofessional care team meetings in real time. As a private college of pharmacy, there was a need to engage external partners to increase ambulatory care experiential learning opportunities, especially those in an interprofessional care team environment. Thus, the partnership was designed to meet the needs of both parties.

Through the partnership, the college of pharmacy provided two days/week of a faculty pharmacist services, which included the preparation for and participation in weekly interprofessional care team meetings and chart reviews, offering drug utilization recommendations and formulary review. As the partnership grew, additional services were provided by the college based on the needs of the organization. These services included addressing periodic drug information requests from medical staff regarding hospice and palliative care topics, as well as leading regular staff educational programs on specific drug topics.

Within the APPE, students received education on the use of medications for patients receiving palliative and end of life care. This included establishing treatment goals, assessing patient response, and appropriate symptom monitoring within a palliative symptom management approach. Learners gained experiences with several common disease states affecting this population including dementia, chronic obstructive pulmonary disease (COPD), heart failure, pain management, bowel regimens, and inappropriate/high risk medication use in the elderly. Learners were also involved in weekly topic discussions including a review during the first week regarding general polypharmacy and deprescribing in advanced age. Weekly topics followed, such as more specific disease state overviews, which included a focus on aging populations and deprescribing within a disease state when prognosis is less than time needed to benefit for certain therapies. Learners assessed the risks and benefits of a potential therapy for its ability to provide immediate symptom relief or acute harm while factoring in formulary and cost considerations. A highlight of the experience was interactions with physicians, nurses, social workers, and other care providers in an interprofessional approach to address drug therapy issues that arise including logistical, economic, and therapeutic considerations. Along with the pharmacist preceptor, who was a faculty member trained in geriatric care, students attended in-person weekly interprofessional care team meetings at the hospice organization and provided real-time interventions during patient care plan discussions.

Due to logistical capacity, only a select number of care teams within the hospice organization included pharmacy students and the faculty preceptor at the time of data collection. After more than two years since implementation, there was a need to evaluate the efficacy of this partnership from learner, organizational, and patient outcome perspectives. This study utilized a mixed-methods approach, including multiple data sources and perspectives, for a holistic evaluation of the partnership. This study was approved by the university's institutional review board.

\section{Quantitative}

A retrospective chart review assessed polypharmacy differences in de-identified hospice patients on two regional teams at time intervals consistent with group interdisciplinary meetings throughout their hospice benefit. Patients with a primary terminal diagnosis of non-Alzheimer's dementia admitted from June 2018 to June 2019 were included for comparison. Non-Alzheimer's dementia represents the largest patient group currently receiving hospice benefits, and on average, the longest length of stay (LOS) in hospice, providing additional time to allow for interdisciplinary meetings, discussion, and deprescribing interventions. ${ }^{20,21}$ Group 1 was an interprofessional care team including academic-practice partnership student pharmacists and the pharmacy preceptor during weekly interdisciplinary group meetings. Group 2 did not include a pharmacist and represented the standard of care for teams. For Group 2, all pharmacy services were completed by the offsite, mail order pharmacy.

No patients reviewed were excluded from this study, regardless of age or short LOS on hospice active patient census. Total number of medications were recorded at admission, week 4 , week 6 , and a week between 7-12, depending on survival, to assess the impact of routine pharmacist deprescribing interventions during team meetings between these intervals versus normal care. Specific medication classes were not included in the deprescribing analysis attributable to an expedited timeline of data collection and change in electronic health record platform. The authors hypothesized care teams, including academic-practice partnership student pharmacists and a pharmacy preceptor, would have less polypharmacy burden in patients than teams without pharmacists. To determine overall impact on deprescribing medications, the groups were compared regarding baseline criteria, as well as total medications at each interval to determine a difference between the two groups using the Student $t$-test, with a null hypothesis of no difference in mean medications. Alpha was set at 0.05 . 


\section{Qualitative}

A purposive sample of 22 care team members, who interacted with APPE students and preceptors, were invited to participate in semi-structured key informant interviews with members of the research team to document perceptions of student pharmacists and preceptor contributions to the care team and organization. Interviews were conducted August - September 2019. A semi-structured interview guide was developed following Gillham's recommended approach. ${ }^{22}$ The guide was used during all interviews to enhance consistency and fidelity, with questions centering on partnership history, team member impact, patient impact, and organizational impact. All participants signed informed consent agreements. Interviews were facilitated by two pharmacy student research assistants trained in qualitative data collection. Interviews were held in private rooms at the hospice organization facilities, lasted approximately 30 minutes, were captured through a digital audio recording device, and transcribed verbatim by an outside agency.

All (11) students who completed the APPE from July 2019 through June 2020 were assigned reflective writings and were asked to consent for their responses to be used for research purposes. Prompts structured around Kirkpatrick's evaluation framework asked learners to focus their reflections on APPE. ${ }^{23}$ Specifically, the students were asked to complete a 3-4 page reflection responding to the following prompts: 1 ) Describe your reaction to the rotation.; 2) What did you learn during the rotation?; 3) How did your behaviors, attitudes, or perceptions of end of life care change throughout the rotation?; 4) What was your role on the care team; 5) If applicable, describe how your contributions led to any improvements for patients. Provide specific case examples, excluding identifiers.

Qualitative data were analyzed using grounded theory, as this approach is useful in developing a general explanation of processes, actions, or interactions, such as the dynamics in the interprofessional care teams. ${ }^{24,25}$ Upon completion of all interviews, transcripts were checked for accuracy and uploaded into NVivo 12 (QSR International, Cambridge, MA). All student reflective essays were de-identified, numbered, and also uploaded to NVivo. A primary coder, who had training and extensive experience in qualitative research methods and analysis, conducted open, line-by-line coding as data were collected. Axial coding explored relationships and connections between initial codes. As themes and relationships began to emerge, consultation with the broader research team allowed for confirmation and further refinement of codes and relationships through an iterative process. Constant comparison was employed throughout the data analysis process, allowing for a back-and-forth interplay across all data sources as additional codes emerged from newly collected data sources. Conceptual categories were identified and grouped into properties linked to these relationships. Matrix coding queries were conducted to triangulate code sources. Emergent themes were compiled with supporting text from interviews and reflections.

\section{Results}

\section{Quantitative}

Table 1 presents patient characteristics and medications at baseline (admission to hospice care). There were no statistically significant differences between groups.

Table 1. Group Characteristics at Baseline (Admission)

\begin{tabular}{|l|c|c|c|}
\hline & Group 1 & Group 2 & $\boldsymbol{p}$ value $^{\mathbf{a}}$ \\
\hline Total Patients & 81 & 97 & \\
\hline Mean Age & 85.4 & 84.7 & .58 \\
\hline Mean Medications & 9.9 & 10.6 & .41 \\
\hline
\end{tabular}

a Student $t$-test used to determine significance, defined as $p<.05$ Group 1=partnership team including pharmacy preceptor and students

Group 2=no pharmacist on team (control)

Table 2 presents a comparison of changes in mean medications between groups over time. Group 1 showed a decrease in mean medications from admission at each data point. Group 2 did not demonstrate a decrease in mean medications, and saw an increase in mean medications from admission to the last data point. The addition of a pharmacist on a care team significantly decreased the average number of medications at two separate data points, week 4 and weeks 7-12. The difference in average medications between Group 1, with a pharmacist, and Group 2, without a pharmacist, were statistically significant. However, the week 6 average difference was not statistically significant.

\begin{tabular}{|l|c|c|c|}
\hline \multicolumn{4}{|c|}{ Table 2. Changes in Mean Medications Over Time } \\
\hline & Group 1 & Group 2 & $\boldsymbol{p ~ v a l u e ~}^{\mathbf{a}}$ \\
\hline Admission & 9.9 & 10.6 & .41 \\
\hline Week 4 & 8.7 & 10.5 & $<.001$ \\
\hline Week 6 & 9.1 & 10.4 & .18 \\
\hline Week 7-12 & 8.6 & 11.4 & .005 \\
\hline
\end{tabular}

a Student $t$-test used to determine significance, defined as $p<.05$ Group1=partnership team including pharmacy preceptor and students

Group2=no pharmacist on team (control)

\section{Qualitative}

Fifteen (68\%) care team members participated in interviews, including 5 nurses, 2 physicians, 2 social workers, 2 chaplains, 1 nurse practitioner, 1 nurse supervisor, 1 volunteer manager, and 1 team assistant. Only 6 student reflections (54.5\%) were included in this study. Four reflections were excluded from students who completed the rotation during the months of March - June 2020, as the COVID-19 pandemic significantly changed the APPE learning environment. In addition, one student declined for their data to be used for research purposes. 
Through qualitative data analysis, the authors conceptualized a theoretical model illustrating perceptions of pharmacy contributions to care teams in the academic-practice partnership. The model, presented in Figure 1, includes the key constructs of medication expertise, interprofessional collaboration, patient-centered care, and student empowerment. Both non-pharmacist care team members, as well as pharmacy students, identified interprofessional collaboration and patient-centered care within care teams. As students participated and applied medication expertise in team meetings, they felt empowered in their professional roles.
Figure 1. Perceptions of Pharmacy Contributions to Partnership Care Teams

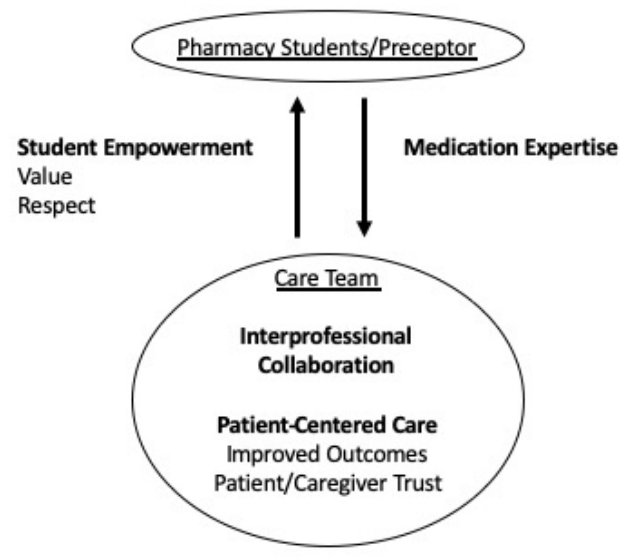

examples of improved patient outcomes through pharmacist recommendations. Interprofessional collaboration was a focal point, as team members acknowledged the educational environment, knowledge sharing, and collaborative decision making that occurs in the partnership. Team members felt better educated on medication regimens, allowing them to better counsel and build trust with patients and their families on medication changes. Finally, team members shared their desire for sustainability and expansion of this care model. the pharmacist allows for non-pharmacy team members to focus on other patient needs. Team members provided

Table 3 provides a summary of conceptual themes that emerged from the stakeholder interviews, indicating the number of interviews, including the theme, total references in all interviews, and representative quotes. Team members had limited prior exposure to pharmacists and expressed newfound appreciation, recognizing pharmacists as medication experts and a valuable resource with the most current medication updates. They shared how delegating medication expertise to

Table 3. Key Informant Interview Conceptual Themes

\begin{tabular}{|l|c|c|c|}
\hline \multicolumn{2}{|c|}{ Table 3. Key Informant Interview Conceptual Themes } \\
\hline Conceptual Theme & $\begin{array}{c}\text { Interviews Including } \\
\text { Theme (N=15) } \\
\text { No. (\%) }\end{array}$ & $\begin{array}{c}\text { Total } \\
\text { References }\end{array}$ & Representative Quote \\
\hline $\begin{array}{l}\text { Pharmacist as Team } \\
\text { Medication Experts }\end{array}$ & 12 (80) & 25 & $\begin{array}{l}\text { [It's] a huge benefit to have somebody who specializes in } \\
\text { medications... it's like where we specialize in palliative care, we } \\
\text { specialize in end of life symptoms, you all specialize in } \\
\text { medications. }\end{array}$ \\
\hline $\begin{array}{l}\text { Improved Patient } \\
\text { Outcomes }\end{array}$ & 11 (73) & 24 & $\begin{array}{l}\text { We had a patient that was falling every single day... We did two } \\
\text { tiny medication adjustments and he never fell again, started } \\
\text { sleeping through the night... It was a medicine he didn't need } \\
\text { anymore. }\end{array}$ \\
\hline $\begin{array}{l}\text { Interprofessional } \\
\text { Collaboration }\end{array}$ & 11 (73) & $\begin{array}{l}\text { [To] just collaborate to see if there's something better, just like } \\
\text { we did today. If there's something better, or what we could } \\
\text { use, or utilize it differently. I think that helps the physician also } \\
\text { just to throw back and forth what would be best for the } \\
\text { patient. }\end{array}$ \\
\hline $\begin{array}{l}\text { Patient/Caregiver } \\
\text { Trust in Medication } \\
\text { Regimens }\end{array}$ & $8(53)$ & 14 & $\begin{array}{l}\text { [T]he families feel more comfortable knowing that... I ran it by a } \\
\text { pharmacist too. We double checked and we both feel that this } \\
\text { will be a good fit for their husband, their wife. That's been } \\
\text { helpful. }\end{array}$ \\
\hline $\begin{array}{l}\text { Desire for } \\
\text { Sustainability }\end{array}$ & $13(87)$ & $\begin{array}{l}\text { I think that overall, it's been a huge value add...I would like to see } \\
\text { that expand...People are jealous of us because we do have a } \\
\text { pharmacist in team with us and we have ready access. }\end{array}$ \\
\hline
\end{tabular}


All student reflective essays included the following themes: teamwork, respect, value, and patient-centered care. One student summarized:

"When I would get questions during team that I could answer with confidence, I felt like I was very valuable to the meetings. At the end of the day, I felt like we were respected by the team and they realized that we are the medication experts."

Another shared:

"I enjoyed sitting in on the conversations about patients and hearing stories from nurses, social workers and chaplains. Each person in the team brought a unique view to the table... I enjoyed being able to listen to their approaches. I was able to gain insight on how these patients lived each day, which ultimately helped me understand how medications played a role in their care."

\section{Discussion}

The results from this study build on the existing literature on academic practice partnerships in pharmacy education that provide rich descriptions of formalized partnerships, but did not pair that information with patient or student learning outcomes resulting from the partnership. ${ }^{26-28}$ With data from multiple assessment points, this study demonstrates clear benefits from the partnership agreement that advance the missions of both organizations. In examining patient medication outcomes, the addition of a pharmacist on an interprofessional team decreased the average amount of medications at week 4 and week 7-12. However, the week 6 average difference in total number of medications was not statistically significant. This could be attributed to lack of deprescribing opportunities between week 4 and week 6 with only one interprofessional team meeting taking place between this time interval. This short-term increase at week 6 could also be attributed to medications added for acute symptom management with a predefined comfort pack ordered for each patient admitted for hospice services in a home setting. However, the average difference between the groups was approximately 1.5 medications with a trend toward statistical significance, which may be considered clinically significant.

Qualitative findings suggest this partnership model is mutually beneficial to both hospice team members and student pharmacists. Care team members clearly articulated examples of value-added contributions of pharmacists on the care team, expressing desire for partnership expansion and sustainability. Learners were able to share their knowledge as medication experts, building confidence in their clinical skills and understanding of the pharmacist's role on an interprofessional team. Similar to the findings of Rodgers et al., ${ }^{29}$ students recognized the value that they brought to the practice site. Overall, this APPE appears to serve as a successful example of an academic-practice partnership that promotes and progresses interprofessional practice, falling in line with the AACP Strategic Plan, as well as the Interprofessional Education
Collaborative (IPEC) Competencies for Interprofessional Collaborative Practice. ${ }^{16,30}$ Organizationally, data from this study may provide a rationale for the hospice organization to further invest in pharmacists as part of all care teams. National consensus guidelines recommend the addition of a pharmacist on an interprofessional team to decrease symptom burden, however there is no mandatory reimbursement requirement for a pharmacist's presence on a team. ${ }^{31}$ The addition of pharmacists could lead to an overall decrease in cost and symptom burden in this population by targeted deprescribing. Further studies are needed to examine more comprehensive patient outcomes as well as cost savings within care teams that include pharmacists.

\section{Limitations}

This study has several limitations. Quantitatively, the overall LOS between groups was not assessed, which could have identified patients with better (longer) prognoses at baseline. Primary care provider (PCP) designation was not compared, which may have delayed deprescribing changes in medication orders. Deprescribing was only assessed by total number of medications, without consideration for drug class or specific medications, there was no differentiation between as needed versus routine scheduled medications, and there were no other outcomes assessed. Furthermore, the hospice organization was undergoing a change in electronic health record platform at the time of this study, which expedited the timeline for data collection. The authors recognize that additional information regarding the medication classes deprescribed would improve the ability to extrapolate the potential benefits of deprescribing in other patient populations. Future studies should evaluate deprescribing interventions by drug class, with consideration to evidence-based tools to identify potentially inappropriate prescribing. ${ }^{32}$

Additionally, qualitative components included small sample sizes, and data collection tools were designed specifically to explore this partnership. Thus, qualitative findings discerned through this study are specific to this partnership and are not generalizable. While $68 \%$ of care team members who interacted with student pharmacists participated in the key informant interviews, there was the potential for respondent bias in that the 15 who agreed to participate in the interviews may have felt more favorably toward pharmacy involvement compared to the 7 care team members who declined. Furthermore, the COVID-19 pandemic significantly shifted the learning environment for 4 of the 11 students who completed the APPE during the data collection period, leading to a small sample size for the student reflections.

\section{Conclusion}

This study was a multi-lensed assessment of an interprofessional academic-practice partnership in end of life care. Overall, this study clearly identified mutual benefits for both organizations participating in the partnership that go far beyond measuring the number of increased APPE rotations or 
funding for clinical staffing within partner organizations. Outcomes suggest that the addition of a pharmacist on an interprofessional team decreased the average number of medications in the partner organization's non-Alzheimer's end of life patient population. Interprofessional care team members identified value-added contributions of student pharmacists and the pharmacy preceptor that enhanced team efficiency and patient care. Finally, student pharmacists, who completed the $A P P E$, recognized these contributions and the experience served an exemplar of interprofessional practice. Overall, this study demonstrates the contribution of academic pharmacy partnerships to interprofessional care teams, which may assist other colleges of pharmacy in implementing sustainable learning experiences for students through similar partnership models.

Acknowledgements: The authors thank Mariah Applegate, PharmD and Jena Fisher, PharmD, student research assistants on this project, for their assistance with key informant interview data collection.

\section{Conflict of Interest: None}

Funding/support: This project was funded by the AACP 2019 Scholarship of Teaching and Learning Grant.

\section{References}

1. Herndon CM, Nee D, Atayee RS, et al. ASHP guidelines on the pharmacist's role in palliative and hospice care. Am J Health Syst Pharm. 2016;73(17):13511367. doi:10.2146/ajhp160244

2. Pruskowski J, Arnold R, Skledar SJ. Development of a health-system palliative care clinical pharmacist. Am J Health Syst Pharm. 2017;74(1):e6-e8. doi:10.2146/ajhp160055

3. Scott IA, Hilmer SN, Reeve E, et al. Reducing inappropriate polypharmacy: the process of deprescribing. JAMA Intern Med. 2015;175(5):827834. doi:10.1001/jamainternmed.2015.0324

4. Schenker Y, Park SY, Jeong K, et al. Associations between polypharmacy, symptom burden, and quality of life in patients with advanced, life-limiting illness. J Gen Intern Med. 2019;34(4):559-566. doi:10.1007/s11606-019-04837-7

5. McGrath K, Hajjar ER, Kumar C, Hwang C, Salzman B. Deprescribing: a simple method for reducing polypharmacy. J Fam Pract. 2017;66(7):436-445.

6. Stinson MJ, Gurevitz S, Carrigan A. Deprescribing at the end of life in older patients. JAAPA.

2019;32(7):20-24. doi:10.1097/01.JAA.0000558316.40245.ca
7. Anderson, K, Stowasser, D, Freeman, C, et al. Prescriber barriers and enablers to minimising potentially inappropriate medications in adults: A systematic review and thematic synthesis. BMJ Open. 2014;4(12):e006544. doi:10.1136/bmjopen-2014006544

8. Kua CH, Mak VSL, Huey Lee SW. Health outcomes of deprescribing interventions among older residents in nursing homes: a systematic review and metaanalysis. J Am Med Dir Assoc. 2018;20(3):362372.e11. doi:10.1016/j/jamda.2018.10.026

9. Page AT, Clifford RM, Potter K, et al. The feasibility and the effect of deprescribing in older adults on mortality and health: a systematic review. Br J Clin Pharmacol. 2016;82(3):583-623. doi:10.1111/bcp.12975

10. Foley RA, Hurard LL, Cateau D, et al. Physicians', Nurses' and Pharmacists' Perceptions of Determinants to Deprescribing in Nursing Homes Considering Three Levels of Action: A Qualitative Study. Pharmacy (Basel). 2020;8(1):17. doi:10.3390/pharmacy8010017

11. Mishori R. What needs to change to make deprescribing doable. Fam Pract Manag. 2018;25(3):5-6.

12. Raman-Wilms L, Farrell BJ, Sadowski CA, et al. Deprescribing: an educational imperative. Res Social Adm Pharm. 2019;15(6):790-795. doi:10.1016/j.sapharm.2018.08.011

13. Farrell B, Conklin J, Dolovich L, et al. Deprescribing guidelines: an international symposium on development, implementation, research and health professional education. Res Social Adm Pharm. 2019;15(6):780-789. doi:10.1016/j.sapharm.2018.08.010

14. Pruskowski J, Patel R, Brazeau G. The need for palliative care in pharmacy education. Am J Pharm Educ. 2019;83(5):7410. doi:10.5688/ajpe7410

15. Clark CM, Hejna M, Shao E, et al. Knowledge and attitudes of student pharmacists regarding polypharmacy and deprescribing: a cross-sectional study. Pharmacy (Basel). 2020;8(4):220. doi:10.3390/pharmacy 8040220

16. American Association of Colleges of Pharmacy. Strategic Plan. https://www.aacp.org/article/strategic-plan. Published June 2018. Accessed October 5, 2021.

17. Gubbins PO, Micek ST, Badowski M, et al. Innovation in clinical pharmacy practice and opportunities for academic-practice partnership. Pharmacotherapy. 2014;34(5):e45-54. doi:10.1002/phar.1427 
18. de Bittner MR, Adams AJ, Burns AL, et al. Report of the 2010-2011 professional affairs committee: effective partnership to implement pharmacists' services in team-based, patient-centered healthcare. Am J Pharm Educ. 2011;75(10):S11. doi:10.5688/ajpe7510S11

19. Hall PD, Fish $H, M c B a n e ~ S$, et al. The report of the 2018-2019 professional affairs standing committee: the role of educators in pharmacy practice transformation. Am J Pharm Educ. 2019;83(10):7596. doi:10.5688/ajpe7596

20. Aldridge MD, Canavan M, Cherlin E, et al. Has hospice use changed? 2000-2010 utilization patterns. Med Care. 2015;53(1):95-101. doi:10.1097/MLR.0000000000000256

21. U.S. Centers for Medicare and Medicaid Services. Medicare hospice data. https://www.cms.gov/Medicare/Medicare-Fee-forServicePayment/Hospice/Medicare_Hospice_Data.html. Published April 12, 2013. Accessed October 5, 2021.

22. Gillham B. The Research Interview. New York, NY: Continuum; 2000.

23. Kirkpatrick DL. Evaluating Training Programs: The Four Levels. San Francisco, CA: Berrett-Koehler; 1994.

24. Charmaz K. Constructing Grounded Theory: $A$ Practical Guide through Qualitative Analysis. Los Angeles, CA: Sage; 2006.

25. Creswell JW. Qualitative Inquiry and Research Design: Choosing among Five Approaches ( $2^{\text {nd }}$ ed.). Thousand Oaks, CA: Sage; 2007.
26. Hall RG, Foslein-Nash C, Singh DK, et al. A formalized teaching, practice, and research partnership with the Veterans Affairs North Texas Health Care System: a model for advancing academic partnerships. Am J Pharm Educ. 2009;73(8):141. doi:10.5688/aj7308141

27. Bird ML, Dunn RL, Hagemann TM, Burton ME, Britton $\mathrm{ML}$, St Cyr MB. Collaboration between a college of pharmacy and a for-profit health system at an academic medical center. Am J Health Syst Pharm. 2012;69(13):1150-1156. doi:10.2146/ajhp110550

28. Metzger $\mathrm{N}$, Paciullo $\mathrm{C}$, Chesson $\mathrm{M}$, et al. Unique collaboration between a private college of pharmacy and a private academic health system. Hosp Pharm. 2014;49(7):634-638. doi:10.1310/hpj4907-634

29. Rodgers PT, Fecteau A, Leadon KI. Student perceptions of the value they bring to APPE sites. Curr Pharm Teach Learn. 2019;11(10):1035-1040. doi:10.1016/j.cptl.2019.06.006

30. Interprofessional Education Collaborative. Core Competencies for Interprofessional Collaborative Practice: 2016 Update. Washington, DC: Interprofessional Education Collaborative; 2016.

31. National Consensus Project for Quality Palliative Care. Clinical Practice Guildelines for Quality Palliative Care, 4th ed. Richmond, VA: National Coalition for Hospice and Palliative Care; 2019.

32. Hukins D, Macleod U, Boland JW. Identifying potentially inappropriate prescribing in older people with dementia: a systematic review. Eur J Clin Pharmacol. 2019;75(4):467-481. doi:10.1007/s00228018-02612-x 